\title{
A conservative and well-balanced surface tension model
}

\author{
By Moataz O. Abu-Al-Saud ${ }^{1}$, Stéphane Popinet ${ }^{2}$ and Hamdi A. Tchelepi ${ }^{1}$ \\ 1) Department of Energy Resources Engineering, Stanford University, Stanford, CA 94305, \\ USA \\ 2) Sorbonne Université, Centre National de la Recherche Scientifique, Institut Jean Le \\ Rond $\partial$ 'Alembert, F-75005 Paris, France \\ Email: stephane.popinet@upmc.fr
}

\begin{abstract}
This article describes a new numerical scheme to model surface tension for an interface represented by a level-set function. In contrast with previous schemes, the method conserves fluid momentum and recovers Laplace's equilibrium exactly. It is formally consistent and does not require the introduction of an arbitrary interface thickness, as is classically done when approximating surface-to-volume operators using Dirac functions. Variable surface tension is naturally taken into account by the scheme and accurate solutions are obtained for thermocapillary flows. Application to the Marangoni breakup of an axisymmetric droplet shows that the method is robust also in the case of changes in the interface topology.
\end{abstract}

\section{Introduction}

A wide range of numerical models for interfacial flows rely on an Eulerian description of the velocity, pressure and material property fields. This is natural for interfacial fluid flows that involve very large deformations as well as topological changes (merging and fragmentation). The discrete volumetric representation of fields which results from the Eulerian approach is however not naturally suitable for the accurate representation of surface forces, such as surface tension [23]. A classical "trick" is to formally transform the surface force density into a volumetric force density (a body force) through multiplication by a surface Dirac function. For example, the contribution of surface tension to fluid momentum is usually written

$$
D_{t}(\rho \boldsymbol{u})=-\nabla p+\gamma \kappa \boldsymbol{n} \delta_{s},
$$

with $D_{t}$ the total derivative, $\rho$ the density, $\boldsymbol{u}$ the velocity, $p$ the pressure, $\gamma$ the surface tension coefficient, $\kappa$ the interface curvature, $\boldsymbol{n}$ the unit normal to the interface. The surface Dirac function $\delta_{s}$ is non-zero only on the interface. The surface tension $\gamma \kappa$ is a force per unit area ; it is transformed into a force per unit volume through product with the Dirac function (the inverse of a vanishingly-small length scale).

This volumetric transform is exploited by a range of closely-related numerical models which can be traced back to the original "immersed boundary" method of Peskin [21]. The surface tension force density is rewritten as

$$
\gamma \kappa \boldsymbol{n} \delta_{s}=\gamma \kappa \nabla H_{s},
$$


with $H_{s}$ the interface Heaviside function. Various numerical approximations can be chosen for $H_{s}$. In the Continuum-Surface-Force (CSF) method of Brackbill et al. [3] and subsequent papers $H_{s}=c$, where $c$ is the volume fraction; in levelset methods and in the immersed boundary method of Peskin, as well as some front-tracking implementations, $H_{s}=f(\phi)$ where $\phi$ is the levelset function and $f$ is some smooth approximation of a Heaviside function (e.g. a hyperbolic tangent or cosine function); in the Ghost-FluidMethod $(\mathrm{GFM}), H_{s}=(\operatorname{sign}(\phi)+1) / 2$. These different approximations have a minor influence on the overall scheme and all these CSF-based methods share common properties [23]. In particular, the characteristic interface thickness is always of order $\Delta$, the mesh size, which is consistent with the introduction of this length scale through the Dirac function. Note that this diffusion of the interface over $\Delta$ is also true for the GFM, despite claims that it is a "sharp interface" method, in contrast with the others. As a consequence, all the above methods are formally first-order accurate in space. This follows from detailed analysis of Peskin's scheme by LeVeque \& Li [13] and is independent from the order of accuracy of the curvature $\kappa$.

A very important property explains the success of this class of methods. As first noted by Renardy \& Renardy [25], the equilibrium condition between pressure and surface tension can be written

$$
-\boldsymbol{\nabla} p+\gamma \kappa \boldsymbol{n} \delta_{s}=-\boldsymbol{\nabla} p+\gamma \kappa \boldsymbol{\nabla} H_{s}=0,
$$

which reduces to

$$
\nabla\left(p_{\text {eq }}-\gamma \kappa_{\text {eq }} H_{s}\right)=0
$$

in the case of constant surface tension and curvature. Hence $p_{\text {eq }}=\gamma \kappa_{\text {eq }} H_{s}$, which is just Laplace's relation for a spherical droplet in equilibrium. From a numerical point of view, this well-balancing property holds only if the discrete gradient operator applied to $p$ is identical to that applied to $H_{s}$, otherwise truncation errors may not cancel. Another condition for well-balancing is that the numerical method converges toward a constant curvature estimate, which is far less trivial. Curvature estimation techniques, rather than Heaviside approximations, are indeed the discriminating ingredient within well-balanced CSF methods. Well-balancing is crucial in practice, because interfacial flows often involve small deviations from Laplace equilibria. Any numerical imbalance can thus swamp the true solution. Exact well-balancing was first demonstrated by Popinet [22] for a VolumeOf-Fluid (VOF) method with a Height-Function curvature estimation and has since been obtained also for levelset [1].

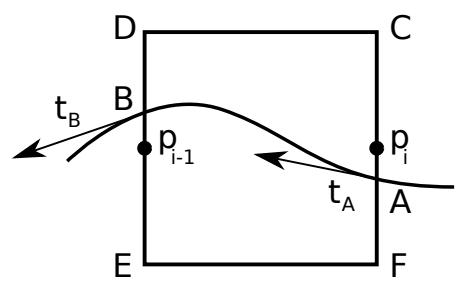

Figure 1. Controle volume and interface tangents.

A discrete surface tension model can also be derived in a very different manner. If one considers the discrete volume $\Omega$ of Figure 1, intersected by an interface, the resultant of the surface tension forces acting on $\Omega$ is simply

$$
\oint_{A}^{B} \gamma d \boldsymbol{t}=\gamma_{B} \boldsymbol{t}_{B}-\gamma_{A} \boldsymbol{t}_{A},
$$


with $\gamma_{A}, \boldsymbol{t}_{A}$ the surface tension coefficient and unit tangent at intersection point $A$ (resp. $B)$. Note that this is mathematically equivalent to the volumetric formulation since we have

$$
\oint_{A}^{B} \gamma d \boldsymbol{t}=\oint_{A}^{B} \gamma \kappa \boldsymbol{n} d s=\int_{\Omega} \gamma \kappa \boldsymbol{n} \delta_{s},
$$

where we used the first Frenet-Serret relation for parametric curves. From a discrete point-of-view this is very different, however. This is because we replaced the estimation of interface curvature, normal and Dirac/Heaviside with the estimation of interface tangents and positions. Furthermore, we avoided the introduction of an arbitrary length scale (the interface thickness) so that the interface is now truly sharp. The order of the method is controlled only by the accuracy with which we describe the interface geometry.

It is obvious from expression (3) that the net force exerted by surface tension on a closed contour/surface is necessarily zero (i.e. surface tension has no effect on the variation of total momentum). This is clearly true for the discrete scheme since the force $\gamma_{A} \boldsymbol{t}_{A}$ acting on a control volume also acts on the neighboring control volume but with the sign reversed. Surface tension is indeed a locally and globally momentum-conserving force (indeed a contact force rather than a body force), which can thus be written

$$
\oint_{A}^{B} \gamma d \boldsymbol{t}=\int_{\Omega} \boldsymbol{\nabla} \cdot \boldsymbol{\sigma}
$$

with $\sigma$ the surface tension stress tensor. This formulation was used in particular by Gueyffier et al. [9] to derive a different model, the Continuum-Surface-Stress (CSS) approximation. Note that the volumetric formulations (1) offer no such guarantee with respect to momentum conservation.

One last significant advantage of the integral formulation (3) is that it naturally includes the tangential stresses due to a variable surface tension (i.e. Marangoni stresses). For volumetric methods, the term $\delta_{s} \boldsymbol{\nabla}_{s} \gamma$ needs to be added, and computing the surface gradient $\nabla_{s}$ accurately is not trivial $[17,27]$.

It is clear that the integral formulation has significant formal and possibly practical advantages over the volumetric formulation. So why is it not more commonly used? A first element of answer is that the simple well-balancing relation (2) is lost, so that it is not obvious whether this method can be well-balanced. A second element is that, up-to-now, it was only applied within a high-order (spline-based) front-tracking interface description framework by Popinet \& Zaleski [24]. While very accurate, this formulation was complex and could not handle changes in interface topology.

In this work, we revisit the integral formulation of Popinet \& Zaleski (1999) coupled this time with a levelset representation of the interface. We seek to evaluate and understand whether the method is well-balanced, clarify its formulation and its practical implementation. The ultimate goal is to obtain a method which is both well-balanced and momentumconserving, in contrast with current schemes.

\section{Numerical scheme}

We develop discrete expressions for the components of the surface tension tensor $\boldsymbol{\sigma}$. To illustrate the principle of the derivation, we consider the control volume for the horizontal velocity/momentum component sketched on Figure 1. Note that the derivation assumes a standard Marker-And-Cell (MAC) staggered discretisation, where the discrete velocity components reside at cell faces and the pressure at cell centers (Figure 2). The method can also be applied to discretisation schemes where the pressure and velocity are collocated, provided the acceleration is expressed at cell faces, which is necessary for well-balancing (see [22] for details). 
As pointed out in Popinet \& Zaleski (1999), we need to consider the pressure and surface tension contributions to the stress tensor simultaneously in order to derive wellbalanced expressions. For the particular case depicted in Figure 1 these contributions to the horizontal momentum can be written as

$$
\begin{aligned}
{\left[\int_{\Omega}-\nabla p+\delta_{s}\left(\gamma \kappa \boldsymbol{n}+\nabla_{s} \gamma\right)\right] \cdot \boldsymbol{x} } & =-\oint_{\partial \Omega} p \boldsymbol{n} \cdot \boldsymbol{x} d s+\oint_{A}^{B} \gamma d \boldsymbol{t} \cdot \boldsymbol{x} \\
& =\oint_{D}^{E} p d s-\oint_{F}^{C} p d s+\left(\gamma_{B} \boldsymbol{t}_{B}-\gamma_{A} \boldsymbol{t}_{A}\right) \cdot \boldsymbol{x}
\end{aligned}
$$

with $\boldsymbol{x}$ the unit vector in the horizontal direction. The pressure along the $C F$ and $D E$ faces is discontinuous due to surface tension and we have jump relations at $A$ and $B$, namely

$$
\begin{aligned}
& {[p]_{A}=\gamma_{A} \kappa_{A},} \\
& {[p]_{B}=\gamma_{B} \kappa_{B},}
\end{aligned}
$$

with $\kappa_{A}$ and $\kappa_{B}$ the corresponding interface curvatures. Assuming piecewise-constant pressures, we get the following first-order-accurate approximations:

$$
\begin{aligned}
\oint_{D}^{E} p d s & \simeq|E B| p_{i-1}+|B D|\left(p_{i-1}-\gamma_{B} \kappa_{B}\right) \\
& =\Delta p_{i-1}-|B D| \gamma_{B} \kappa_{B}, \\
\oint_{F}^{C} p d s & \simeq \Delta p_{i}+|A F| \gamma_{A} \kappa_{A} .
\end{aligned}
$$

The signs for the pressure contributions from surface tension are different in the two expressions. This is because the pressures lie on different sides of the interface. Note that in contrast with Popinet \& Zaleski (1999), we use the curvature of the interface to impose the jump explicitly. Appendix A describes how to compute this jump implicitly.

Expressions (4) and (5) can be generalised if we introduce the surface fractions $s_{i}$ i.e. the relative length of cell faces wetted by one of the two-phases. In our example, they are

$$
\begin{aligned}
s_{i}^{x} & =\frac{|A F|}{\Delta}, \\
s_{i-1}^{x} & =\frac{|B E|}{\Delta} .
\end{aligned}
$$

The general expression for the face-integrated pressure is

$$
\oint_{i} p d s=\Delta p_{i}+\Delta \gamma_{i} \tilde{\kappa}_{i} \begin{cases}s_{i}^{x} & \text { if } s_{i}<1 / 2 \\ s_{i}^{x}-1 & \text { otherwise }\end{cases}
$$

where $\tilde{\kappa}_{i}$ is the curvature of the interface at its intersection with face $i$ (or zero if there is no intersection).

Combining the contributions of surface tension and pressure gradient, we get for the example of Figure 1

$$
\begin{aligned}
\int_{\Omega}-\partial_{x} p+\delta_{s}\left(\gamma \kappa n^{x}+\partial_{s}^{x} \gamma\right)= & -\Delta\left(p_{i}-p_{i-1}\right)-\Delta \gamma_{i} \tilde{\kappa}_{i} s_{i}^{x}+\Delta \gamma_{i-1} \tilde{\kappa}_{i-1}\left(s_{i-1}^{x}-1\right)+ \\
& \gamma_{i-1} t_{i-1}^{x}-\gamma_{i} t_{i}^{x} \\
= & -\Delta\left(p_{i}+\sigma_{i}^{x x}-p_{i-1}-\sigma_{i-1}^{x x}\right)
\end{aligned}
$$

with $t_{i}^{x}$ the horizontal component of the unit tangent to the interface at its intersection with face $i$ (or zero if there is no intersection), and

$$
\sigma_{i}^{x x}=\gamma_{i}\left[\frac{t^{x}}{\Delta}+\tilde{\kappa}\left\{\begin{array}{ll}
s^{x} & \text { if } s^{x}<1 / 2 \\
s^{x}-1 & \text { otherwise }
\end{array}\right]_{i}\right.
$$


In the more general case where the horizontal faces $C D$ and $E F$ may be intersected by the interface, we need to add the contributions from the non-diagonal term of the surface tension stress tensor. There is no pressure contribution to this term since the horizontal component of the normal direction $n^{x}$ is zero for horizontal faces. This horizontal face component can simply be written as

$$
\sigma_{j+1 / 2}^{x y}=\left[\gamma \frac{t^{x}}{\Delta}\right]_{j+1 / 2},
$$

with $t_{j+1 / 2}^{x}$ the horizontal component of the unit tangent to the interface at its intersection with face $j+1 / 2$ (or zero if there is no intersection). The general expression for the horizontal momentum component thus becomes

$$
\int_{\Omega}-\partial_{x} p+\delta_{s}\left(\gamma \kappa n^{x}+\partial_{s}^{x} \gamma\right)=-\Delta\left(p_{i}-p_{i-1}+\sigma_{i}^{x x}-\sigma_{i-1}^{x x}+\sigma_{j+1 / 2}^{x y}-\sigma_{j-1 / 2}^{x y}\right)
$$

The corresponding expressions for the vertical components are simply obtained by rotation of the indices. The final scheme is a consistent discretisation of

$$
\int_{\Omega}-\nabla p+\delta_{s}\left(\gamma \kappa \boldsymbol{n}+\nabla_{s} \gamma\right)=\int_{\Omega}-\nabla \cdot(p \boldsymbol{I}+\boldsymbol{\sigma})
$$

This guarantees that the scheme conserves momentum. Note that, as is usual for the discretisation of stresses on a staggered grid, the stress tensor components are located as depicted in Figure 2.

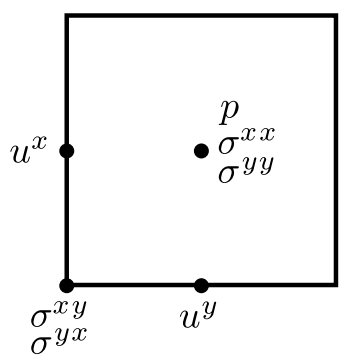

Figure 2. Locations of the discrete velocity, pressure and stress tensor components.

\subsection{Implementation for a levelset interface representation}

We now consider an interface described by the zero-level of a levelset function $\phi$, taken to be an approximation of the signed distance function to the interface. The discrete values of $\phi$ are defined at the same locations as the pressure and diagonal components of the surface tension tensor i.e. for integer indices $i, j$.

\subsubsection{Curvature estimation}

The interface curvature is required to compute the diagonal terms of the surface tension stress tensor. It is estimated at integer locations using a classical finite-difference discretisation of

$$
\kappa_{i, j}=\left(\boldsymbol{\nabla} \cdot \frac{\boldsymbol{\nabla} \phi}{|\boldsymbol{\nabla} \phi|}\right)_{i, j}=\left(\frac{\phi_{x}^{2} \phi_{y y}-2 \phi_{x} \phi_{y} \phi_{x y}+\phi_{y}^{2} \phi_{x x}}{\left(\phi_{x}^{2}+\phi_{y}^{2}\right)^{3 / 2}}\right)_{i, j}
$$


where the derivatives are estimated using central differencing, specifically

$$
\begin{aligned}
\left(\phi_{x}\right)_{i, j} & \simeq \frac{\phi_{i+1, j}-\phi_{i-1, j}}{2 \Delta} \\
\left(\phi_{x x}\right)_{i, j} & \simeq \frac{\phi_{i+1, j}-2 \phi_{i, j}+\phi_{i-1, j}}{\Delta^{2}} \\
\left(\phi_{x y}\right)_{i, j} & \simeq \frac{\phi_{i+1, j+1}-\phi_{i-1, j+1}-\phi_{i+1, j-1}+\phi_{i-1, j-1}}{(2 \Delta)^{2}}
\end{aligned}
$$

\subsubsection{Diagonal terms}

We first consider the diagonal term for the horizontal component of velocity, defined at location $i, j$ i.e. $\sigma_{i, j}^{x x}$. This corresponds to face $F C$ of Figure 1. The corresponding diagonal term for the vertical component of velocity, $\sigma_{i, j}^{y y}$, will be obtained using the same formulation by rotation of the indices and components.

The levelset function is defined at location $i, j$, the middle of face $F C$. Levelset values at corner points $C$ and $F$ can be obtained by simple averaging as

$$
\phi_{i, j+1 / 2}=\frac{\phi_{i, j}+\phi_{i, j+1}}{2}
$$

for point $C$ and respectively for $\phi_{i, j-1 / 2}$ (point $F$ ).

Depending on the relative signs of $\phi_{i, j-1 / 2}, \phi_{i, j}$ and $\phi_{i, j+1 / 2}$, the interface can intersect face $F C$ zero, one or two times. It is important to properly account for all these combinations. This can be simply done by summing up contributions from each configuration. We first describe the approach used for the intersection depicted in Figure 1, for which $\phi_{i, j}$ and $\phi_{i, j-1 / 2}$ have different signs. Following (5) the corresponding contribution to the diagonal term is

$$
\sigma_{i}^{x x}=\gamma_{A}\left[\frac{t_{A}^{x}}{\Delta}+\kappa_{A} s_{A}^{x}\right]
$$

where $t_{A}^{x}$ is the outward, horizontal unit tangent component at point $A, \kappa_{A}$ the interface curvature at point $A$ and $s_{A}^{x}$ the surface fraction. If we assume that $\phi$ is the signed distance function to the interface, the horizontal component of the unit tangent to the interface at point $C$ is the vertical component of the unit normal, i.e.

$$
t_{i, j+1 / 2}^{x}=\left(\phi_{y}\right)_{i, j+1 / 2}=\frac{\phi_{i, j+1}-\phi_{i, j}}{\Delta},
$$

and respectively for the tangent at point $F, t_{i, j-1 / 2}^{x}$. The unit tangent at point $A$ is obtained by linear interpolation between $C$ and $F$ i.e.

$$
t_{A}^{x}=2 \xi t_{i, j-1 / 2}^{x}+(1-2 \xi) \frac{t_{i, j-1 / 2}^{x}+t_{i, j+1 / 2}^{x}}{2},
$$

with

$$
\xi=\frac{\phi_{i, j}}{\phi_{i, j}-\phi_{i, j-1}}
$$

the relative position of the intersection. This gives after simplification

$$
t_{A}^{x}=\frac{1}{\Delta}\left[\frac{\phi_{i, j+1}-\phi_{i, j-1}}{2}-\xi\left(\phi_{i, j-1}-2 \phi_{i, j}+\phi_{i, j+1}\right)\right],
$$


which is simply the centered-difference derivative with a second-order correction. Note that if $\phi$ is the exact signed distance function the normal/tangent to the interface verifies

$$
|\boldsymbol{n}|=|\boldsymbol{\nabla} \phi|=1
$$

however this is not true of the linearly interpolated normal/tangent defined by (7), which should in principle be re-normalised.

The pressure jump $\gamma_{A} \kappa_{A} s_{A}^{x}$ is then computed using the linearly interpolated values

$$
\begin{aligned}
\gamma_{A} & =\gamma_{i, j}+\xi\left(\gamma_{i, j-1}-\gamma_{i, j}\right) \\
\kappa_{A} & =\kappa_{i, j}+\xi\left(\kappa_{i, j-1}-\kappa_{i, j}\right)
\end{aligned}
$$

and

$$
s_{A}^{x}=\frac{1}{2}-\xi .
$$

Note that the interpolation of curvature seems to make little difference on the accuracy of the method so that the approximation $\kappa_{A} \simeq \kappa_{i, j}$ can be used.

Using the above expressions, a simple general algorithm for the computation of $\sigma_{i, j}^{x x}$ is obtained by summing contributions from all possible intersections and taking into account the relevant interface orientations (this explains the absolute value and sign expressions in the last line of the algorithm).

\section{Algorithm 1}

Computation of $\sigma_{i, j}^{x x}$ given $\phi_{i, j}$ the signed distance function, $\kappa_{i, j}$ the corresponding curvature and $\gamma_{i, j}$ the (variable) surface tension coefficient.

$\sigma_{i, j}^{x x} \leftarrow 0$

for $k$ in $-1,1$ :

$$
\text { if } \begin{aligned}
& \phi_{i, j}\left(\phi_{i, j}+\phi_{i, j+k}\right) \leqslant 0: \\
& \xi \leftarrow \frac{\phi_{i, j}}{\phi_{i, j}-\phi_{i, j+k}} \\
& t^{x} \leftarrow \frac{1}{\Delta}\left[\frac{\phi_{i, j+1}-\phi_{i, j-1}}{2}+k \xi\left(\phi_{i, j-1}-2 \phi_{i, j}+\phi_{i, j+1}\right)\right] \\
& \kappa \leftarrow \kappa_{i, j}+\xi\left(\kappa_{i, j+k}-\kappa_{i, j}\right) \text { or simply } \kappa_{i, j} \\
& \gamma \leftarrow \gamma_{i, j}+\xi\left(\gamma_{i, j+k}-\gamma_{i, j}\right) \\
& \sigma_{i, j}^{x x} \leftarrow \sigma_{i, j}^{x x}+\gamma\left[\frac{\left|t^{x}\right|}{\Delta}-\operatorname{sign}\left(\phi_{i, j}\right) \kappa\left(\frac{1}{2}-\xi\right)\right]
\end{aligned}
$$

\subsubsection{Off-diagonal terms}

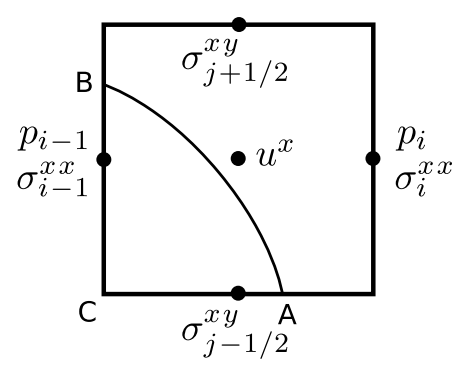

Figure 3. A circular interface intersecting a control volume for the horizontal component of velocity. 
The off-diagonal term $\sigma_{i-1 / 2, j-1 / 2}^{x y}$ (see Figure 3 ) is simpler to compute since the levelset function can only define a single intersection point ( $A$ on Figure 3 ) on face $[i-1$, $j-1 / 2: i, j-1 / 2]$. Furthermore there is no pressure jump contribution to this term. Applying the same reasoning as for the diagonal term then gives the following simple algorithm.

\section{Algorithm 2}

Computation of $\sigma_{i-1 / 2, j-1 / 2}^{x y}$ given $\phi_{i, j}$ the signed distance function and $\gamma_{i, j}$ the (variable) surface tension coefficient.

if $\left(\phi_{i-1, j}+\phi_{i-1, j-1}\right)\left(\phi_{i, j}+\phi_{i, j-1}\right)>0:$

else :

$$
\sigma_{i-1 / 2, j-1 / 2}^{x y} \leftarrow 0
$$

$$
\begin{aligned}
\xi & \leftarrow \frac{\phi_{i-1, j}+\phi_{i-1, j-1}}{\phi_{i-1, j}+\phi_{i-1, j-1}-\phi_{i, j}-\phi_{i, j-1}} \\
t^{x} & \leftarrow \frac{1}{\Delta}\left[\phi_{i-1, j}-\phi_{i-1, j-1}+\xi\left(\phi_{i, j}-\phi_{i-1, j}+\phi_{i-1, j-1}-\phi_{i, j-1}\right)\right] \\
\gamma & \leftarrow \frac{1}{2}\left[\gamma_{i-1, j}+\gamma_{i-1, j-1}+\xi\left(\gamma_{i, j}-\gamma_{i-1, j}-\gamma_{i-1, j-1}+\gamma_{i, j-1}\right)\right] \\
\sigma_{i-1 / 2, j-1 / 2}^{x y} & \leftarrow-\gamma \operatorname{sign}\left(\phi_{i, j}+\phi_{i, j-1}\right) \frac{t^{x}}{\Delta}
\end{aligned}
$$

The corresponding $\sigma_{i-1 / 2, j-1 / 2}^{y x}$ component (the discrete tensor is not symmetric) is obtained from the same algorithm by rotation of the indices.

\subsection{Brief description of the Navier-Stokes solver}

We solve the incompressible variable-density Navier-Stokes equations

$$
\begin{aligned}
\partial_{t} \rho+\boldsymbol{u} \cdot \boldsymbol{\nabla} \rho & =0 \\
\rho\left(\partial_{t} \boldsymbol{u}+\boldsymbol{u} \cdot \boldsymbol{\nabla} \boldsymbol{u}\right) & =\boldsymbol{\nabla} \cdot\left[\mu\left(\boldsymbol{\nabla} \boldsymbol{u}+\boldsymbol{\nabla}^{T} \boldsymbol{u}\right)\right]-\boldsymbol{\nabla} p+\boldsymbol{\nabla} \cdot \boldsymbol{\sigma} \\
\boldsymbol{\nabla} \cdot \boldsymbol{u} & =0
\end{aligned}
$$

The interface kinematics are described by the level-set advection equation:

$$
\frac{\partial \phi}{\partial t}+\boldsymbol{u} \cdot \nabla \phi=0
$$

The different fluid viscosities and densities are determined based on the level-set function:

$$
\mu(\phi)=\mu_{1}+\left(\mu_{2}-\mu_{1}\right) H(\phi),
$$

and

$$
\rho(\phi)=\rho_{1}+\left(\rho_{2}-\rho_{1}\right) H(\phi),
$$

where the subscripts ${ }_{1}$ and ${ }_{2}$ denote the fluid phases, and $H(\phi)$ is the smooth approximation of the Heaviside function

$$
H(\phi)=\frac{1}{2}\left[1-\operatorname{erf}\left(\frac{\phi}{\epsilon}\right)\right],
$$

where erf is the error-function and $\epsilon$ is the width of the interface transition, set to 1.5 times the grid size. 
The conservative surface tension (CST) force in Eq. 8 is computed through the divergence of the surface tension stress tensor as elaborated in Section 2.1.

To discretize the Navier-Stokes equations, the structured Marker And Cell (MAC) method is employed. The velocity vector field $\boldsymbol{u}$ is defined at the grid-cell faces, and the scalar fields $(p, \mu, \rho$, and $\phi)$ are defined at the grid-cell centers (Figure 2). The continuity and momentum equations are solved using the projection method [5]. The advection of the level-set function, Eq. 9, is discretized using a fifth-order WENO scheme in space [11] and the forward-Euler scheme in time. The surface tension contribution to the momentum in Eq. 8 is taken into account when the intermediate velocity $\boldsymbol{u}^{*}$ is computed. This is the first step in the projection method, which is as follows:

$$
\boldsymbol{u}^{*}=\boldsymbol{u}^{n}+\Delta t\left[-(\boldsymbol{u} \cdot \boldsymbol{\nabla}) \boldsymbol{u}+\boldsymbol{\nabla} \cdot\left(\mu\left(\boldsymbol{\nabla} \boldsymbol{u}+\boldsymbol{\nabla} \boldsymbol{u}^{T}\right)\right)+\boldsymbol{\nabla} \cdot \boldsymbol{\sigma}\right]^{n},
$$

The second step in the projection method is the Poisson equation:

$$
\boldsymbol{\nabla} \cdot\left(\frac{\boldsymbol{\nabla} p^{n+1}}{\rho^{n+1}}\right)=\frac{\boldsymbol{\nabla} \cdot \boldsymbol{u}^{*}}{\Delta t}
$$

Finally, the divergence-free velocity is estimated by the following correction step:

$$
\boldsymbol{u}^{n+1}=\boldsymbol{u}^{*}-\Delta t \frac{\nabla p^{n+1}}{\rho^{n+1}} .
$$

In the test cases and examples discussed in this article, the timestep is set according to the classical stability condition for the explicit discretisation of the surface tension term [3]

$$
\Delta t<\sqrt{\frac{\left(\rho_{1}+\rho_{2}\right) \Delta^{3}}{4 \pi \gamma}}
$$

with a safety prefactor varying between 0.5 and 0.8 . The influence of the timestep has been studied and shown to have a negligible impact on the results.

Due to velocity gradients in the flow field, the level-set field has to be reinitialized to maintain it as a signed distance field (which verifies $|\nabla \phi|=1$ ). This is particularly important in the present context since we assumed that this property is verified when estimating the surface tension tensor in section 2.1. The Hamilton-Jacobi (HJ) PDE-based reinitialization with high-order discretization scheme is used in this work. To minimize the artificial displacement of the interface and maintain accurate curvature and tangent computations, the subcell fix approach that takes the interface location into consideration is used [26]. The HJ PDE reinitialization equation is

$$
\frac{\partial \phi}{\partial \tau}+S\left(\phi_{o}\right)(|\nabla \phi|-1)=0
$$

where $S\left(\phi_{o}\right)=\phi_{o} / \sqrt{\phi_{o}^{2}+|\nabla \phi|^{2} \Delta^{2}}$. For level-set values smaller than the grid size $\left(\left|\phi_{o}\right| \leq\right.$ $\Delta)$, the subcell third-order ENO scheme proposed in [7] is used to evaluate the derivatives in the Godunov fluxes. To find the subcell distances required for the grid cells near the zero level-set, quadratic interpolation (following the approach in [18]) is used. When $\left|\phi_{o}\right|>\Delta$, the fifth-order WENO scheme [11] is used to discretize the level-set derivatives. For the temporal discretization, the forward-Euler scheme proposed in [18] is used. As for the number of HJ-PDE iterations, the strategy in [14] is used, since it has been found to provide a good balance between accurate level-set field and computational efficiency. In this method, the reinitialization process is applied when

$$
\||\nabla \phi|-1\|_{L_{1}(B)}>(\Delta)^{n},
$$


where $L_{1}(B)$ is the $L_{1}$ error-norm of the level-set deviation from the Euclidean distance property in the narrow region $B$. In the simulations below, the width of the region $B$ around the fluid interface is $B=\{|\phi|<6 \Delta\}$ which is wide enough to compute the interface geometric properties as well as advect accurately the level-set field while avoiding large levelset gradients. Based on trial and error, the exponent $n$ is chosen to be 2, which was found to be a good balance between accuracy and computational efficiency. With this exponent the curvature converges to first-order even when the interface is advected or deformed.

\section{Test cases}

\subsection{Stationary droplet}

For a static droplet inside a closed domain, we measure the spurious currents as the simulation proceeds in time. Convergence of the spurious currents to machine precision indicates a balance between the pressure jump across the interface and the surface tension force (Laplace balance). In the CSF formulation, it is well known that exact equilibrium between the pressure and the surface tension force can be achieved in the level-set without reinitialization, Volume-of-Fluid, and front tracking methods [1, 22, 2]. However, the wellbalanced property has not been previously demonstrated for a momentum-conserving surface tension force formulation. Figure 4 demonstrates that with our new conservative surface tension method, the maximum velocity converges to zero within machine precision for different grid sizes. The parameters used in this case can be characterized by the Laplace dimensionless number, which is a Reynolds number based on the visco-capillary velocity $\gamma / \mu$ :

$$
\mathrm{La}=\frac{\rho \gamma D}{\mu^{2}},
$$

where $D$ is the droplet diameter. In this example $\mathrm{La}=600$. The evolution of the maximum velocity

$$
\mathrm{Ca}_{\max }=\frac{\|\boldsymbol{u}\|_{\infty} \mu}{\gamma},
$$

as a function of the dimensionless "viscous" time

$$
t^{\star}=t \frac{\mu}{\rho D^{2}},
$$

is illustrated in Figure 4. The initial numerical error in the estimation of interface geometric properties leads to initial spurious currents which decay exponentially due to viscosity on a timescale comparable to the viscous dissipation timescale $D^{2} / \mu$. The initial amplitude depends on the spatial resolution as expected and all the simulations converge toward zero to within machine accuracy, irrespective of spatial resolution. The spurious current behavior is similar to the one observed in the CSF formulation [22]. For the given grid resolutions, the initial velocity error is small such that the level-set field is slightly distorted 
from the signed distance property. The level-set field distortion stays below the threshold defined by Eq. 12 therefore there is no need to reinitialize in this test case.

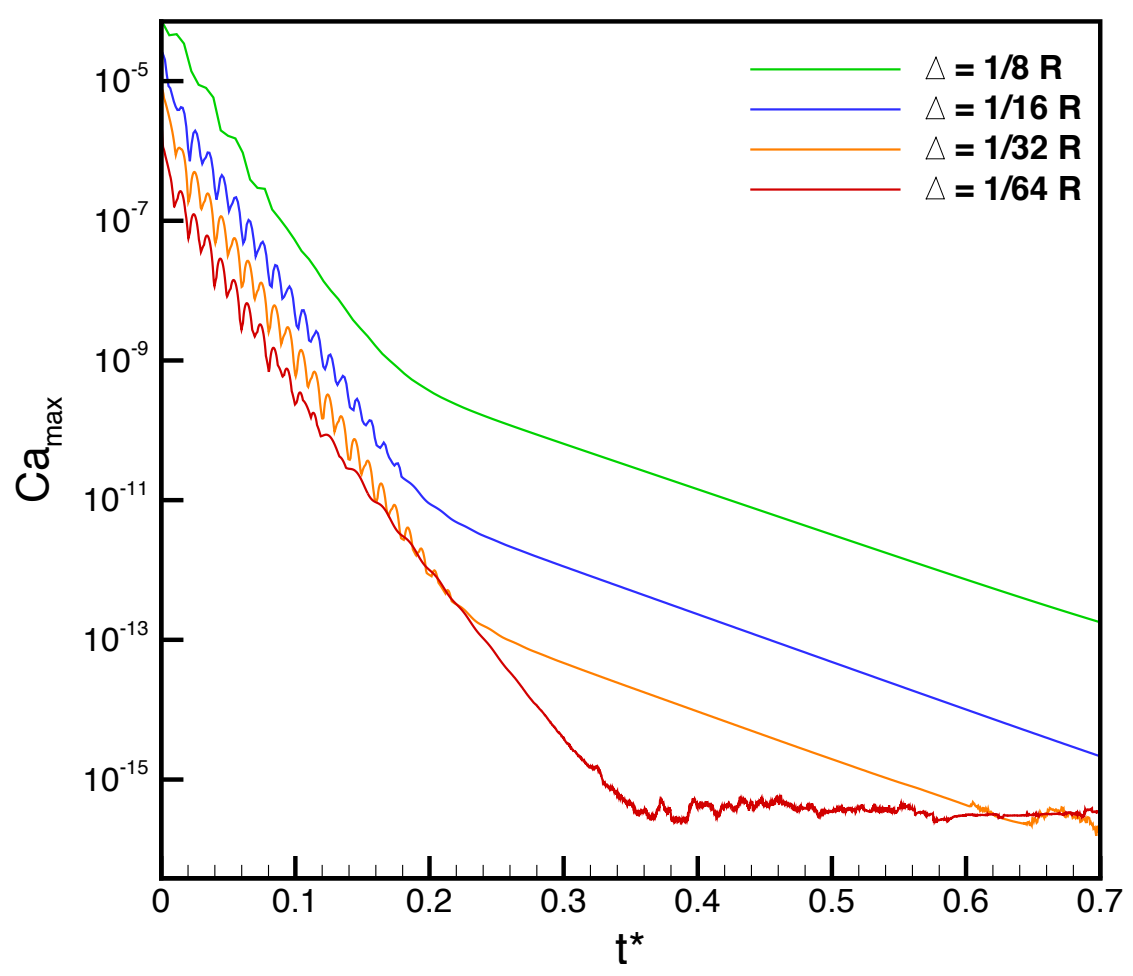

Figure 4. Evolution of the maximum velocity $\mathrm{Ca}_{\max }=\|\boldsymbol{u}\|_{\infty} \mu / \gamma$ as a function of the dimensionless time $t^{\star}=t \mu / \rho D^{2}$, for the spatial resolutions indicated in the legend.

If the reinitialization process is enforced once per timestep, the equilibrium solution is not reached, as already demonstrated for the classical levelset scheme by [1]. Table 1 shows the steady state value of $\mathrm{Ca}_{\max }\left(\right.$ at $\left.t^{\star}=1\right)$ for different grid resolutions. Note that $\mathrm{Ca}_{\max }$ converges with grid refinement. The amplitudes obtained are consistent with those reported by [1], Figure 5, for the LS-CCSF and LS-SSF methods.

\begin{tabular}{|c|c|}
\hline$\Delta$ & $\mathrm{Ca}_{\max }$ \\
\hline $1 / 16 R$ & $4.8 \times 10^{-5}$ \\
\hline $1 / 32 R$ & $1.4 \times 10^{-5}$ \\
\hline $1 / 64 R$ & $4.7 \times 10^{-6}$ \\
\hline
\end{tabular}

Table 1. The maximum steady-state velocity $\mathrm{Ca}_{\max }=\|\boldsymbol{u}\|_{\infty} \mu / \gamma$ for the static droplet with enforced reinitialization at different spatial resolutions.

It is interesting to consider the stationary pressure field obtained at equilibrium. In the case of the well-balanced CSF method the equilibrium pressure field is simply

$$
p=\gamma \tilde{\kappa} H+\text { constant }
$$

where $H$ is the approximation of the Heaviside function used by the method, and $\tilde{\kappa}$ is a spatially-constant numerical estimate of the curvature, which converges toward the exact curvature with spatial resolution (see $[22,23]$ for a detailed discussion). 
For the CST formulation, a simple relation such as (14) does not exist, however we observe constant pressures on either sides of the interface almost everywhere. Figure 5 illustrates the equilibrium pressure distribution obtained for a spatial resolution $\Delta=R / 16$ and $t^{\star}=0.87$, for which $\mathrm{Ca}_{\max }<10^{-15}$. Only the blue cells in Figure 5(b) have values which differ from either the constant inside or outside pressures. These deviations are due to numerical errors in the subcell distances and unit tangents which then violate the equilibrium relations discussed in appendix B. We have checked that if the exact subcell distances and unit tangents are imposed the exact Laplace sharp jump is recovered.

Remarkably, when linear approximations of unit tangents are used, well-balancing (i.e. spurious velocities convergence to machine precision) is obtained, while some pressure values do not obey the Laplace jump. Figure 6 shows the case where the pressure $p_{i, j}$ differs from $p_{i-1, j}, p_{i-1, j-1}$, and $p_{i, j-1}$. This case does not happen in the CSF formulation because the surface tension in CSF does not contribute to the $x$-momentum cell $\rho u_{i-1 / 2, j}^{x}$ or the the $y$-momentum $\rho u_{i, j-1 / 2}^{y}$. In contrast, the CST model includes a surface tension contribution as the interface intersects both momentum cells. As a result, the pressure $p_{i, j}$ differs from the surrounding values within the fluid phase.

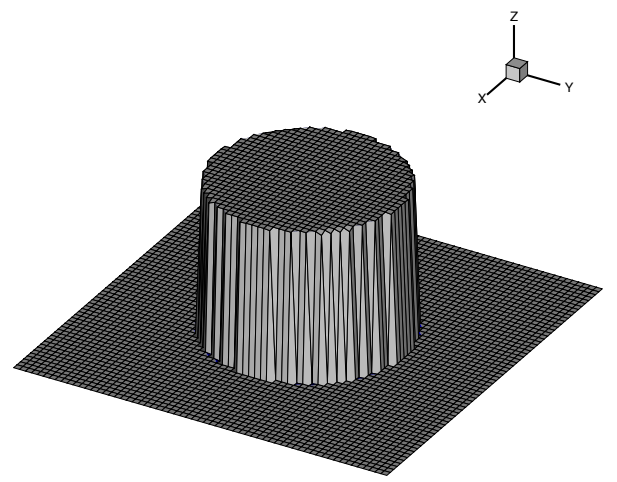

(a)

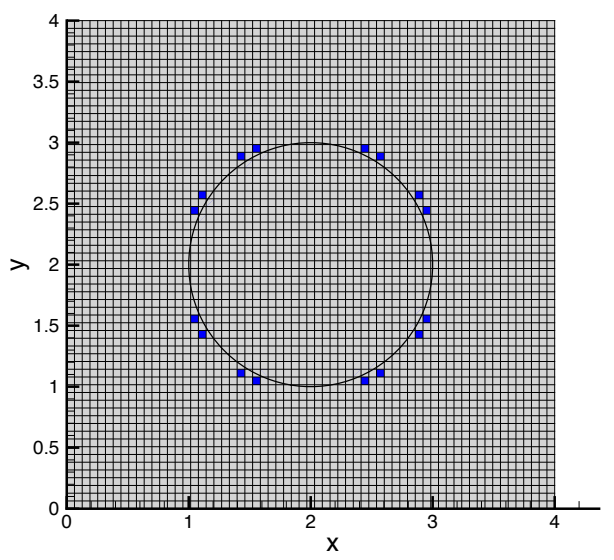

(b)

Figure 5. The pressure distribution at equilibrium for the conservative surface tension (CST) model for $\mathrm{La}=600$ and $\Delta=1 / 16 \mathrm{R}$. (a) pressure field. (b) deviations from the exact Laplace jump.

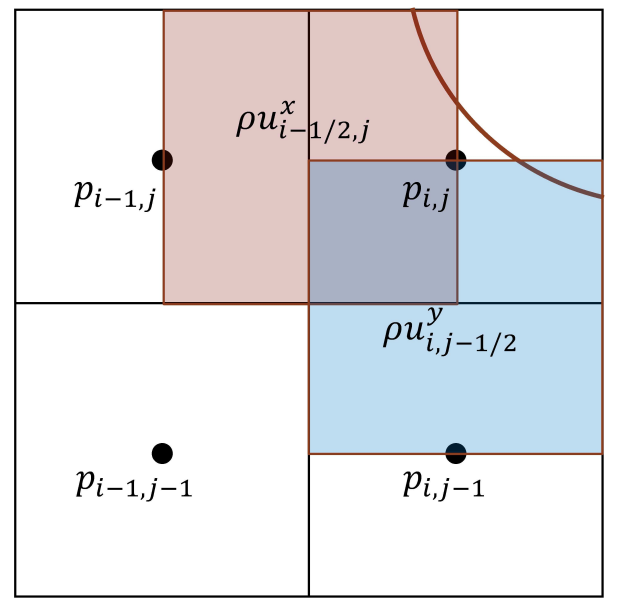

Figure 6. The surface tension contribution to the momentum for the case where the pressure field is not uniform within the fluid phase. 
The accuracy of the numerical equilibrium solution can be estimated using the maximum error on the pressure jump

$$
\frac{\left\|[p]_{\text {comp }}-[p]_{\text {exact }}\right\|_{\infty}}{[p]_{\text {exact }}}=\frac{\left\|\kappa_{\text {comp }}-\kappa_{\text {exact }}\right\|_{\infty}}{\kappa_{\text {exact }}} .
$$

The evolution with spatial resolution of this error is displayed in Figure 7 for the CST and the CSF models. For CSF, the pressure jump is constant spatially, as required by the discrete well-balancing relation (2). Convergence is thus identical in all norms and is governed only by the accuracy of the curvature estimation. This leads to second-order convergence for our scheme.

For the CST model, the error is dominated by the few points where the jump is not constant (blue squares in Figure 5.b). This maximum error converges at less than firstorder rate (red dots in Figure 7). Note however that this maximum error is always small (less than $1 \%$ ) and that other error norms are much smaller (the $L_{1}$-norm relative error is smaller than $10^{-4}$ for $\Delta=1 / 64 R$ ). The $L_{1}$-norm errors are similar to CSF.

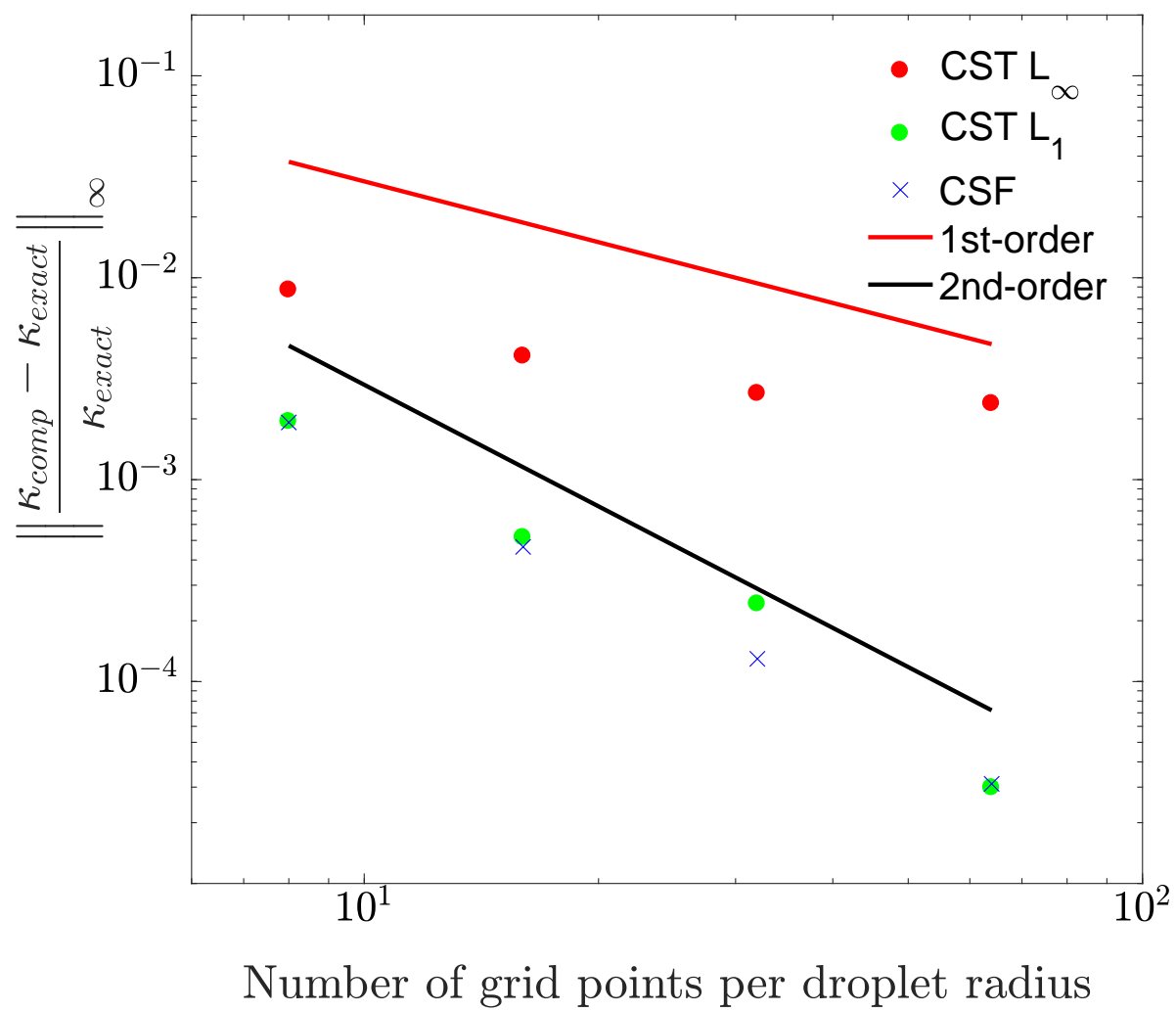

Figure 7. The $L_{\infty}$ and $L_{1}$ errors in the curvature as functions of the grid resolution for the conservative surface tension (CST) and CSF models. The red (resp. black) lines indicate a firstorder (resp. second-order) rate of convergence. 


\subsection{Translating droplet}

When the droplet is advected by a constant periodic velocity field, the spurious currents are due to the coupling between interface advection errors and surface tension errors. The Laplace balance is still the exact solution in the frame of reference of the drop. This test case, initially proposed in [22], has been studied by Abadie et al. [1] for CSF formulations coupled with both VOF and levelset interface representations. We repeat this case with the CST integral formulation. For simplicity, we choose the vertical velocity as a measure of the error, for which the reference solution is zero. Figure 8 shows the $L_{\infty}$ norm of $u^{y}$ scaled by $\gamma / \mu$ for several grid resolutions. The dimensionless time $t^{\star}$ is scaled by $D / U$. The Laplace number is $\mathrm{La}=600$. The imposed horizontal velocity $U$ corresponds to a capillary number $\mathrm{Ca}=\mu U / \gamma=5 \times 10^{-5}$. The $L_{\infty}$ norm of the spurious currents converges with grid refinement. For a grid size of $\Delta=1 / 16 R$ or smaller, the numerical method can simulate flows for capillary numbers down to $\mathrm{Ca}=10^{-5}$. The proposed method is also able to simulate flows of $\mathrm{Ca}=10^{-6}$ for $\Delta=1 / 64 \mathrm{R}$ without introducing errors greater than the imposed flow velocity.

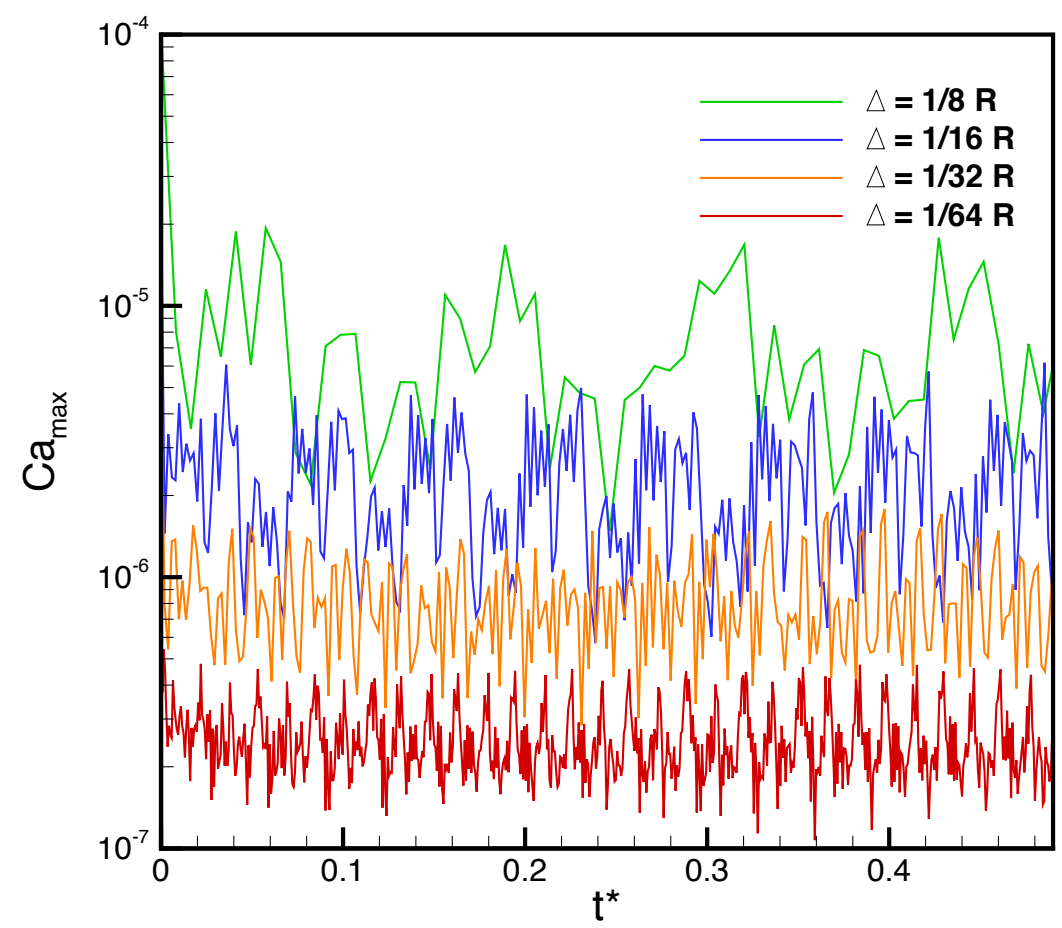

Figure 8. The spurious currents for the moving droplet case at $\mathrm{Ca}=\mu U / \gamma=5 \times 10^{-5}$ for different grid resolutions. $\mathrm{Ca}_{\max }=\left\|u^{y}\right\|_{\infty} \mu / \gamma$ indicates the magnitude of the spurious currents, and $t^{\star}=t U / D$ is the dimensionless time.

Table 2 shows both the $L_{\infty}$-norm and $L_{1}$-norm of $\mathrm{Ca}_{\max }$. The $L_{\infty}$-norm is the maximum 
value of $\mathrm{Ca}_{\max }$ throughout the simulation time, and the $L_{1}$-norm is the average $\mathrm{Ca}_{\max }$ over the period of time where $\mathrm{Ca}$ max oscillates. The oscillation period of $\mathrm{Ca}_{\max }$ corresponds to the droplet movement across one grid cell.

\begin{tabular}{|l|l|l|}
\hline$\Delta$ & $\left\|\mathrm{Ca}_{\max }\right\|_{\infty}$ & $\left\|\mathrm{Ca}_{\max }\right\|_{1}$ \\
\hline $1 / 8 R$ & $1.7 \times 10^{-5}$ & $9.0 \times 10^{-6}$ \\
\hline $1 / 16 R$ & $4.5 \times 10^{-6}$ & $2.2 \times 10^{-6}$ \\
\hline $1 / 32 R$ & $1.6 \times 10^{-6}$ & $8.9 \times 10^{-7}$ \\
\hline $1 / 64 R$ & $4.6 \times 10^{-7}$ & $3.0 \times 10^{-7}$ \\
\hline Order & 1.74 & 1.64 \\
\hline
\end{tabular}

Table 2. The norms of the amplitude of spurious currents for the moving droplet case at $\mathrm{Ca}=$ $\mu U / \gamma=5 \times 10^{-5}$ for different grid resolutions. The last row indicates the rate of convergence.

We now compare the CST model with the CSF model as well as with the results reported in [1]. The Weber number $\mathrm{We}=\rho U^{2} D / \gamma$ is chosen to be 0.4 and the Laplace number is $\mathrm{La}=250$. Figure 9 shows the magnitude of spurious currents as a function of the dimensionless time $t^{\star}=t U / D$ for the grid resolution $\Delta=1 / 12.8 R$. The amplitude of spurious currents for the CST method is larger than for CSF but remains small. The amplitudes for both methods are smaller than $10^{-4}$ which compares favourably with the results reported by [1], Figure 8(a). Our CSF implementation with linearly interpolated curvature is similar to the LS-SSF method in [1].

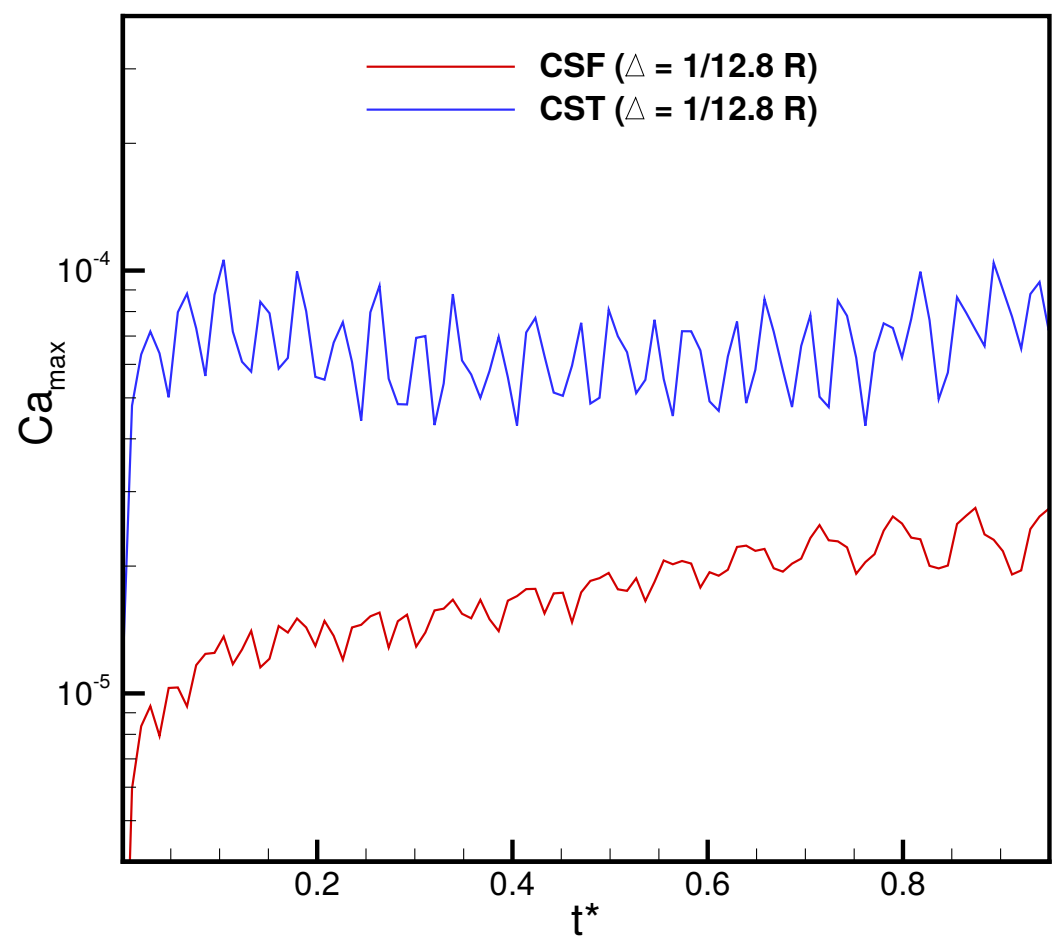

Figure 9. The spurious currents for the moving droplet case at $\mathrm{We}=0.4$ and $\mathrm{La}=250$ for the CST and CSF formulations. $\mathrm{Ca}_{\max }=\left\|u^{y}\right\|_{\infty} \mu / \gamma$ indicates the magnitude of the spurious currents, and $t^{\star}=t(U / D)$ is the dimensionless time. 
Figure 10 illustrates the effect of reinitialization when it is carried out once per timestep for the translating droplet case at $\Delta=1 / 12.8 R$. The spurious currents increase slightly but the effect is marginal which shows that most of the curvature disturbance is caused by errors in transport rather than redistancing.

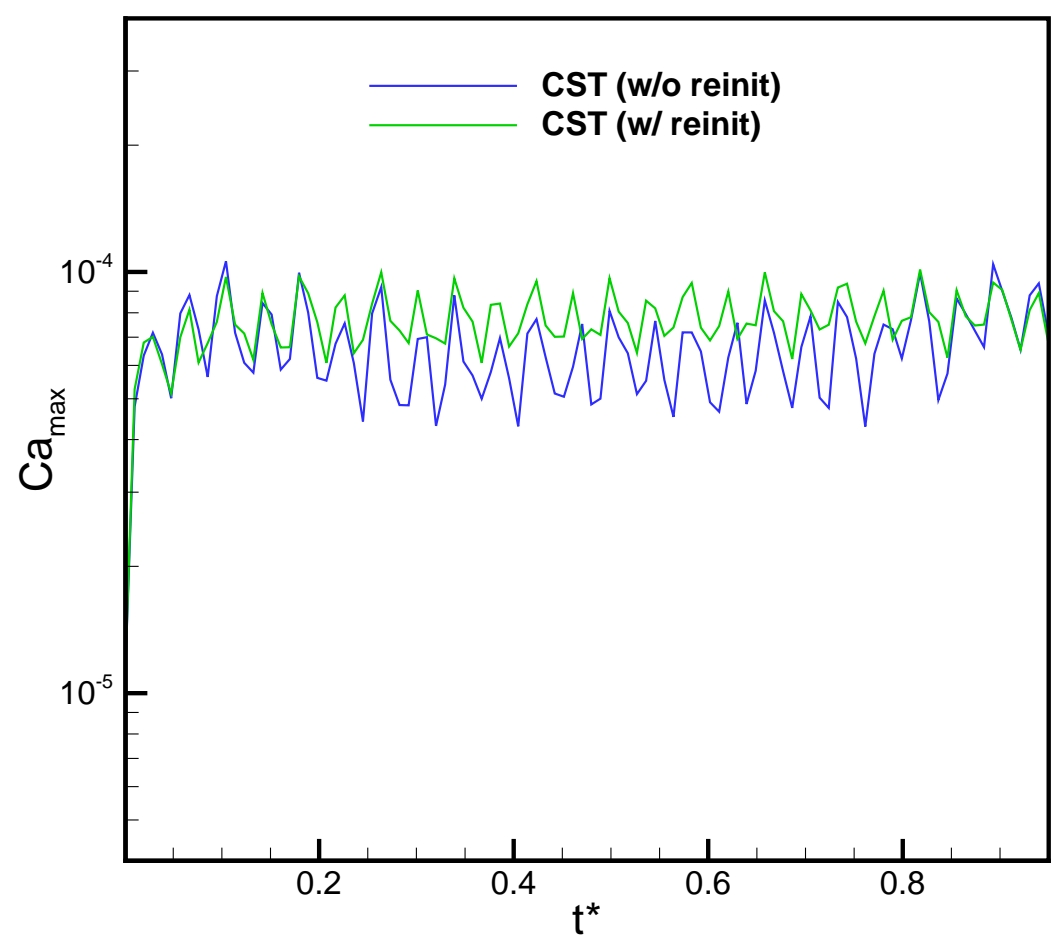

Figure 10. The effect of reinitialization on the amplitude of spurious currents for the moving droplet case at $\mathrm{We}=0.4$ and $\mathrm{La}=250$ for the CST formulation. $\mathrm{Ca}_{\max }=\left\|u^{y}\right\|_{\infty} \mu / \gamma$ indicates the magnitude of the spurious currents, and $t^{\star}=t(U / D)$ is the dimensionless time. The grid size is $\Delta=1 / 12.8 R$. 


\subsection{Oscillating droplet}

We now test the dynamics of the fluid interface by perturbing slightly the initial circular droplet. This interface perturbation causes the droplet to oscillate, due to a physical imbalance between the pressure field and the surface tension force. Lamb [16] derived an analytical solution for the frequency of a slightly perturbed droplet in a vacuum. For a $2 \mathrm{D}$ droplet, the oscillation frequency of the second mode $\omega_{2}$ is $\sqrt{6 \gamma / \rho R^{3}}$. Figure 11 shows the amplitude of the oscillation when the droplet diameter is perturbed by a factor of 1.04 relative to equilibrium. The Laplace number is $\mathrm{La}=20,000$. The viscosity and density ratio between the droplet and its surrounding are 1/1000. The proposed method agrees with the theoretical oscillation frequency. Table 3 and Figure 12 show the frequency error with respect to grid resolution. Second-order convergence is obtained. Level-set reinitialization is carried out according to (12).

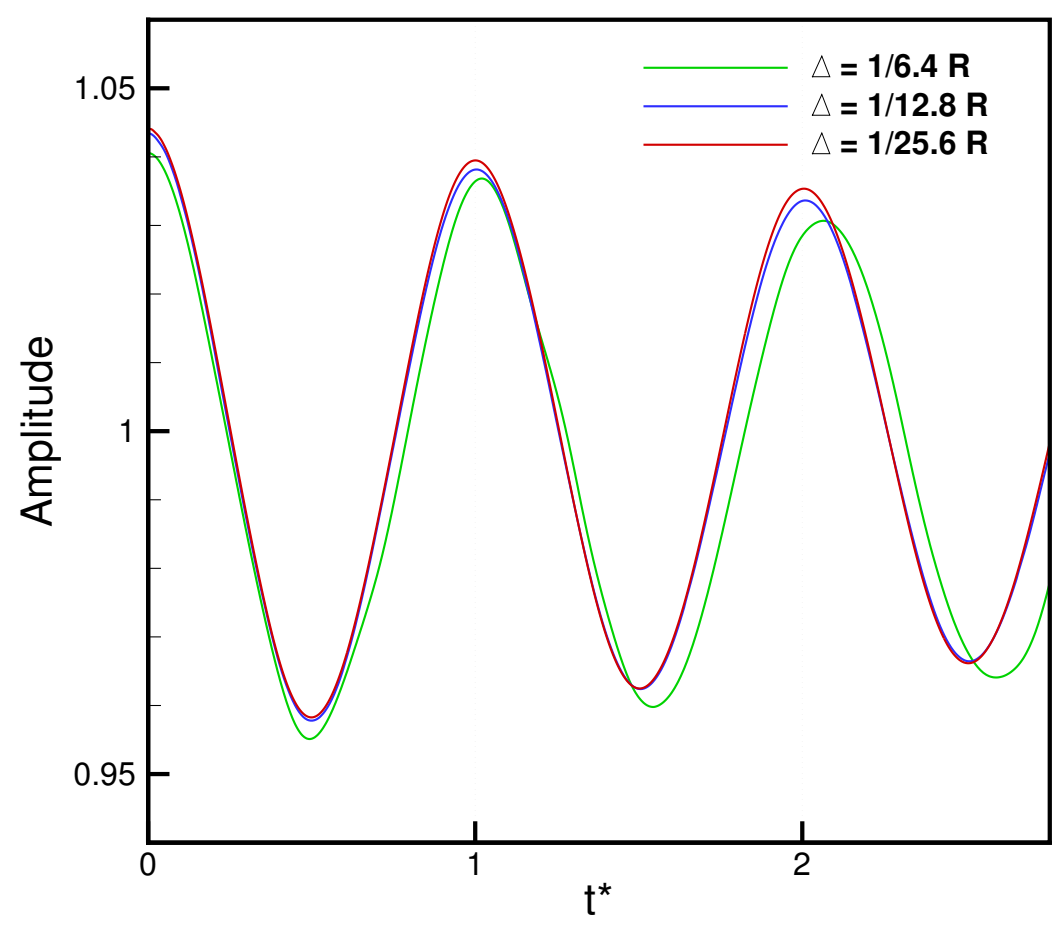

Figure 11. The amplitude of the horizontal droplet diameter vs. dimensionless time $t^{\star}=t \omega_{2} /$ $(2 \pi)$ at different grid resolutions.

\begin{tabular}{|c|c|}
\hline$\Delta$ & Error $\%$ \\
\hline $1 / 6.4 \mathrm{R}$ & 2.05 \\
\hline $1 / 12.8 \mathrm{R}$ & 0.37 \\
\hline $1 / 25.6 \mathrm{R}$ & 0.09 \\
\hline
\end{tabular}

Table 3. The frequency error for the oscillating droplet case for different grid resolutions. 


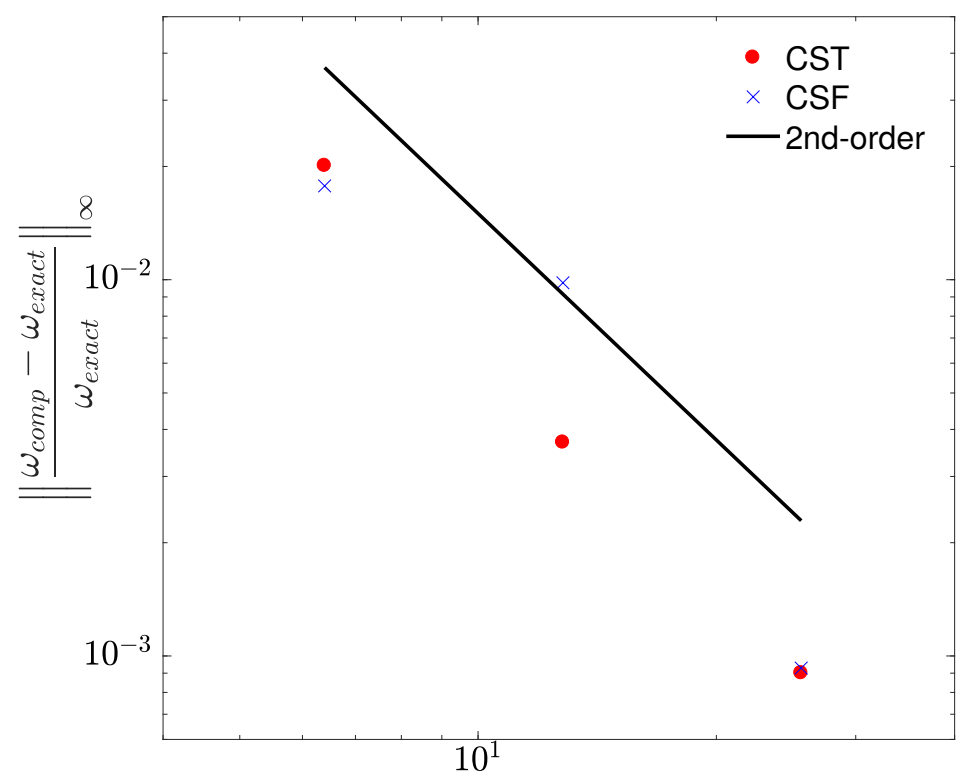

Number of grid points per droplet radius

Figure 12. Convergence of the oscillating frequency with spatial resolution for the CST and CSF models. The black line indicates second-order convergence.

\subsection{Marangoni-induced translation due to a temperature gradient}

In this test case, we evaluate the quality of the Marangoni tangential stress evaluation which is trivially formulated within the integral surface tension model. The surface tension coefficient is a linear function of temperature:

$$
\gamma=\gamma_{0}+\gamma_{T}\left(T-T_{0}\right) .
$$

This surface tension temperature dependency induces a thermo-capillary motion caused by the non-zero tangential Marangoni stresses. The droplet migrates from the cold region to the hot region. Young et al. [28] derived an expression for the velocity of a spherical droplet due to thermocapillarity for small $\mathrm{Re}$ and $\mathrm{Ca}$, which is:

$$
U_{\text {drop }}=\frac{-2}{\left(2+3 \mu_{\text {drop }} / \mu_{\text {bulk }}\right)\left(2+c_{\text {drop }} / c_{\text {bulk }}\right)} \frac{\gamma_{T} R \nabla T}{\mu_{\text {bulk }}},
$$

where $R$ is the droplet radius, $\gamma_{T}$ is the surface tension temperature coefficient, and $c$ is the thermal conductivity. The Reynolds and capillary numbers are defined as $\operatorname{Re}=\rho_{\text {bulk }} U R /$ $\mu_{\text {bulk }}$ and $\mathrm{Ca}=\mu_{\text {bulk }} U / \gamma_{0}$, where the velocity scale is defined as $U=\gamma_{T} R \nabla T / \mu_{\text {bulk }}$.

Figure 13 shows the ratio between the computed axisymmetric droplet velocity and the theoretical solution for different grid sizes. The domain size is $16 R \times 16 R$, which has been found to be large enough to minimize the influence of boundaries on the droplet velocity. The average droplet velocity is computed as follows:

$$
u_{\text {drop }}=\frac{\int H(\phi) u_{c}^{x} d V}{\int H(\phi) d V}=\frac{\Sigma_{c v} \psi_{c v} u_{c}^{x}}{\Sigma_{c v} \psi_{c v}},
$$

where $u_{c}^{x}$ is the horizontal velocity defined at the grid center through arithmetic averaging, and $\psi_{c v}$ is the volume fraction enclosed by the droplet. The volume fraction is computed 
by reconstructing the droplet interface from the level-set information. The interface reconstruction is second-order, where each computational cell is divided into simplexes and the interface intersection points are determined through linear interpolation [6]. The relevant flow parameters are $\mathrm{Re}=0.066$ and $\mathrm{Ca}=0.066$. The density ratio is one. This configuration has been studied in $[20,27,10,4]$. The time is scaled by $\mu_{b} / \gamma_{T} \nabla T$. The ratio of the thermal conductivity $c_{\text {drop }} / c_{\text {bulk }}$ is unity, which means that the temperature distribution in the domain remains the same. The predicted droplet velocity converges quickly with refinement and matches the theoretical solution almost exactly for $\Delta \leqslant 1 / 16 \mathrm{R}$ (Table 4). The results improve on previous studies based on refined level-set grid (RLSG) [4], and Volume-Of-Fluid (VOF)/Height Function method [27]. The quality of the solution, and in particular the absence of spurious velocities, can also be assessed qualitatively through Figure 14 .

The effect of the viscosity ratio has been studied. Figure 13(b) shows the evolution of the droplet velocity when the droplet viscosity is halved. The droplet velocity increases as expected (Table 4) and matches the theoretical solution almost exactly for $\Delta=1 / 32 \mathrm{R}$. In this test case, level-set re-initialization is required to have accurate results because the velocity gradients are significant. The re-initialization process is applied as specified by (12).

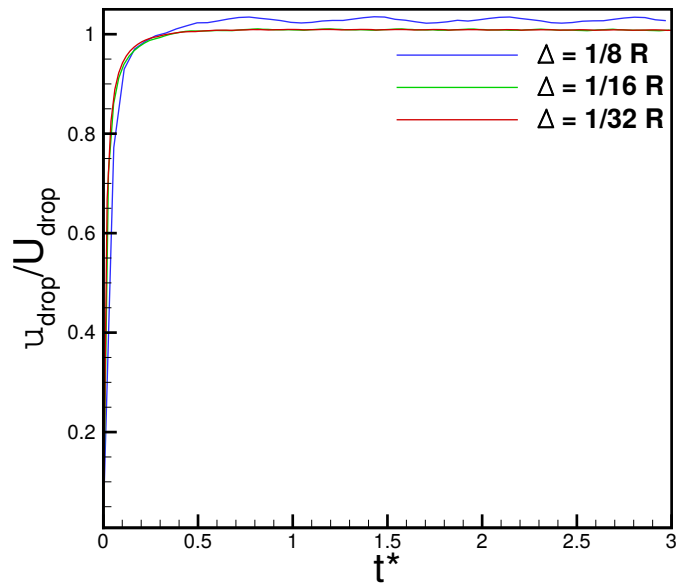

(a) $\mu_{\text {drop }} / \mu_{\text {bulk }}=1$

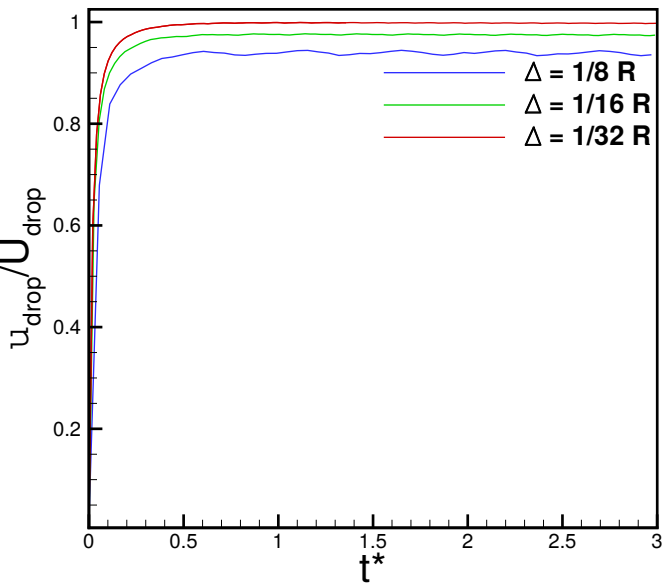

(b) $\mu_{\text {drop }} / \mu_{\text {bulk }}=0.5$

Figure 13. The ratio of the computed axisymmetric droplet velocity $u_{\text {drop }}$ over the theoretical solution $U_{\text {drop }}$ as a function of $t^{\star}=t\left(\gamma_{T} \nabla T / \mu_{b}\right)$ for different viscosity ratios, for $\operatorname{Re}=0.066$ and $\mathrm{Ca}=0.066$.

\begin{tabular}{|l|l|l|l|l|}
\hline & \multicolumn{2}{|c|}{$\mu_{\text {drop }} / \mu_{\text {bulk }}=1$} & \multicolumn{2}{c|}{$\mu_{\text {drop }} / \mu_{\text {bulk }}=0.5$} \\
\hline$\Delta$ & $u_{\text {drop }} / U$ & $U_{\text {drop }} / U$ & $u_{\text {drop }} / U$ & $U_{\text {drop }} / U$ \\
\hline $1 / 8 R$ & 0.1378 & 0.13333 & 0.1785 & 0.19048 \\
\hline $1 / 16 R$ & 0.1347 & 0.13333 & 0.1860 & 0.19048 \\
\hline $1 / 32 R$ & 0.1345 & 0.13333 & 0.1890 & 0.19048 \\
\hline Order & \multicolumn{2}{|c|}{1.74} & \multicolumn{2}{c|}{1.5} \\
\hline
\end{tabular}

Table 4. The computed terminal velocity $u_{\mathrm{drop}} / U$ and the theoretical droplet velocity $U_{\mathrm{drop}} / U$ (eq. 16) for different viscosity ratios, with $U=\gamma_{T} R \nabla T / \mu_{\text {bulk }}$ the characteristic velocity scale, $\mathrm{Re}=0.066$ and $\mathrm{Ca}=0.066$. The last row indicates the rate of convergence. 


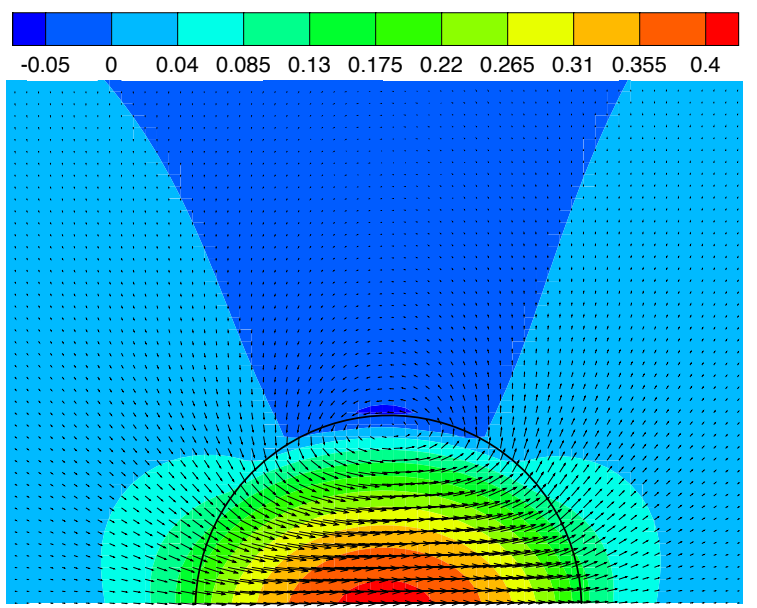

Figure 14. Velocity field and magnitude of the horizontal velocity component $\left(u_{x} / U\right)$ for Marangoni-induced translation of a drop. $\mathrm{Re}=0.066, \mathrm{Ca}=0.066$ and $\mu_{\mathrm{drop}} / \mu_{\text {bulk }}=1$.

\section{Bubble break-up due to variable surface tension}

We now apply the conservative surface tension (CST) method to a more complex case that includes interface break-up. Interface break-up is a key mechanism in many two-phase flow applications such as spray atomization [8], oil trapping in porous media [15], and foam generation [12]. In this example we study the break-up of a bubble due to variable surface tension. This has important potential applications, for example as a way to control bubble distribution in microfluidics devices [19].

Figure 15 shows the surface tension distribution described by the following equation:

$$
\gamma\left(x^{\star}\right)=\gamma_{0} \max \left(1-1.25\left|x^{\star}-x_{0}^{\star}\right|, 0.1\right)
$$

where $x_{0}^{\star}$ is the location of the center of the bubble and $x^{\star}=x / R$, with $R$ the bubble radius. The threshold 0.1 prevents the surface tension value from reaching zero. The domain size is $3.5 R \times 0.75 R$. The Laplace number, $\mathrm{La}=\rho_{\text {bubble }} \gamma_{0} D / \mu_{\text {bubble, }}^{2}$ is 3.44 . The viscosity and density ratios are $1 / 25$, where the bubble has the lower viscosity and density values.
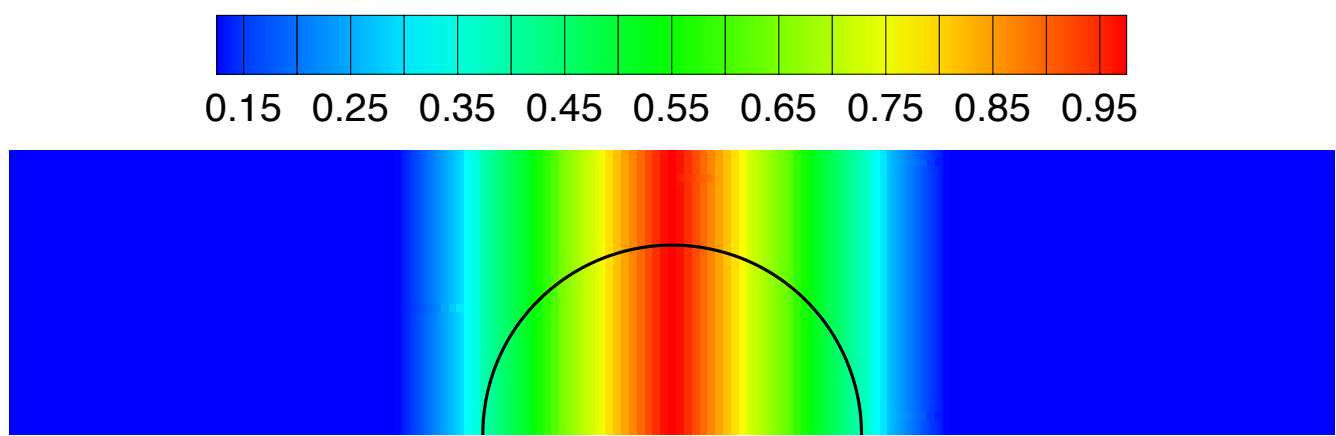

Figure 15. The surface tension distribution (normalized by $\gamma_{0}$ ) and bubble interface. The domain is axisymmetric and the axis of symmetry is aligned with the bottom boundary. 


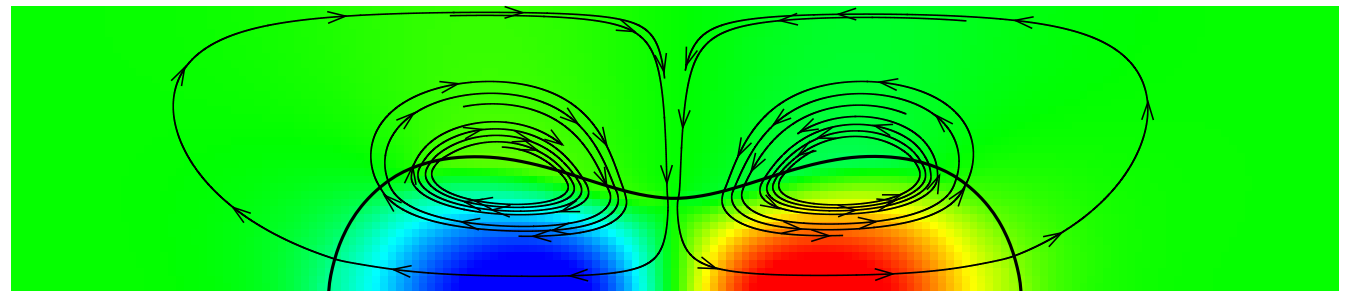

Figure 16. The axisymmetric flow streamlines and the horizontal velocity field.

Figure 16 shows the streamlines of the flow driven by variable surface tension. Flow takes place along the bubble interface from the low surface-tension regions at the bubble extremities to the high surface-tension region in the bubble center. This transient regime driven by variable surface tension is coupled with the classical Rayleigh-Plateau instability and leads to bubble break-up.

Figure 17 illustrates the process of the bubble shape snap-off at different times. The bubble break-up time converges with grid refinement as shown in Figure 18 and Table 5. The results confirm that the proposed method can simulate the complex process of interface break-up due to variable surface tension.

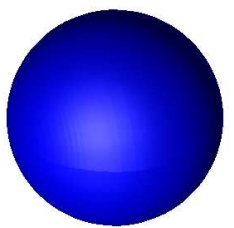

(a) $t^{*}=0$

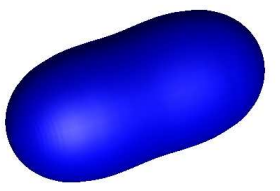

(d) $t^{*}=5.2$

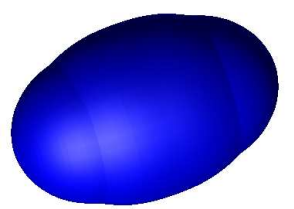

(b) $t^{*}=2.22$

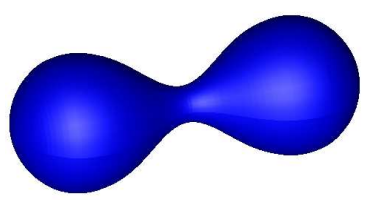

(e) $t^{*}=8.75$

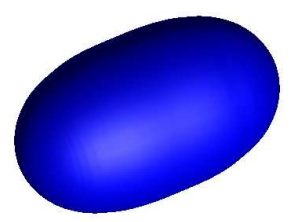

(c) $t^{*}=3.70$
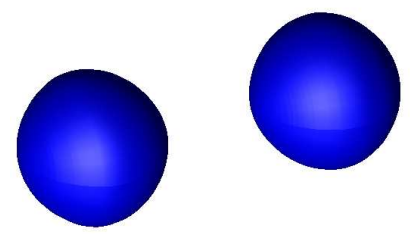

(f) $t^{*}=10$

Figure 17. Bubble snap-off due to variable surface tension at different times, with $t^{*}=t / T_{\sigma}$ and $T_{\sigma}=\sqrt{\rho D^{3} / \gamma_{0}}$. 


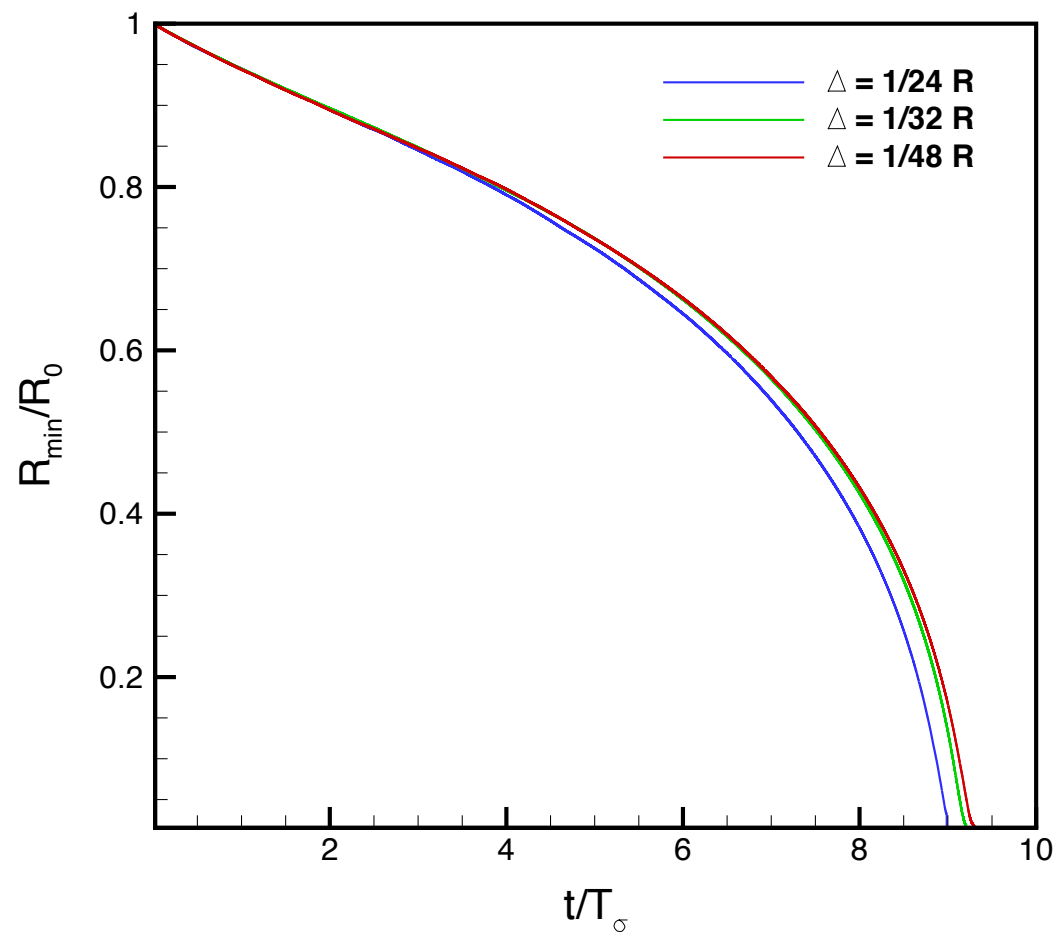

Figure 18. Convergence with grid resolution of the time evolution of the normalized minimum droplet radius.

\begin{tabular}{|c|c|}
\hline$\Delta$ & $t^{\star}$ \\
\hline $1 / 16 R$ & 8.95 \\
\hline $1 / 32 R$ & 9.2 \\
\hline $1 / 64 R$ & 9.3 \\
\hline
\end{tabular}

Table 5. Convergence with grid resolution of the droplet break-up times.

\section{Conclusion}

We have developed a new conservative surface tension (CST) method inspired from the integral formulation of Popinet and Zaleski [24], in the level-set framework. The scheme relies on a finite-volume discretisation of the divergence of the surface tension stress tensor, and thus guarantees strict conservation of momentum. In contrast with earlier work on conservative schemes for surface tension [24, 9], we show that the method is well-balanced and competitive with the best available schemes in term of accuracy of equilibrium solutions. The formal consistency of the integral formulation, which does not require approximate surface-to-volume operators, leads to a true sharp interfacial force representation which naturally includes Marangoni stresses. Classical thermocapillary motion is thus accurately modelled in a straightforward manner.

Several avenues for further development are open. An obvious extension is the application of the method to static and moving contact lines. This is trivial to implement since the contact angle directly gives the tangent at the contact point required to compute the corresponding component of the surface tension tensor. 
The extension to three dimensions is also of great interest. The formalism presented here is applicable in three dimensions but requires the approximation of line integrals rather than point-value estimates (contact lines instead of contact points). The fact that the $2 \mathrm{D}$ formulation is simple (it is fully described by Algorithms 1 and 2) gives confidence that a comparatively simple formulation can also be found in 3D. Whether this also leads to well-balancing in $3 \mathrm{D}$ is more difficult to guess, since our 2D study has shown that some choices of approximations do not lead to well-balancing (linearity of the operators seems to be required). This remains to be clarified.

Finally, the generalisation of the method to other interface representations, for example Volume-Of-Fluid combined with Height-Functions, would also be of great practical interest.

\section{Appendix A Implicit jumps}

We do not need to assume that the pressure jumps due to surface tension at the intersection points are known (which requires the estimation of interface curvature). The pressure jumps can be estimated using either a time-explicit approximation (as was done in Popinet \& Zaleski, 1999) or while inverting the Poisson equation for the pressure. In either case, the expression for the diagonal component of the surface tension tensor needs to be modified to

$$
\sigma_{i}^{x x}=\left[\gamma \frac{t^{x}}{\Delta}+\left\{\begin{array}{ll}
s^{x}\left(p_{j-1}-p_{j}\right) & \text { if } s^{x}<1 / 2 \\
\left(s^{x}-1\right)\left(p_{j}-p_{j+1}\right) & \text { otherwise }
\end{array}\right]_{i}\right.
$$

Note that this formulation could be advantageous since it may relax some of the consistency constraints between tangent and curvature estimations required for well-balancing. We have not yet pursued this question further, however.

\section{Appendix B Well-balancing}

Let us consider the configuration illustrated in Figure 3. At equilibrium, the pressure and surface tension terms must balance, which can be written

$$
p_{i}-p_{i-1}+\sigma_{i}^{x x}-\sigma_{i-1}^{x x}+\sigma_{j+1 / 2}^{x y}-\sigma_{j-1 / 2}^{x y}=0,
$$

where $p_{i}=p_{i-1}$ (since the pressure jumps are taken into account in the surface tension tensor) and $\sigma_{i}^{x x}=\sigma_{j+1 / 2}^{x y}=0$ (since the interface does not intersect the corresponding faces of the control volume). Using the discrete expressions for the components of the stress tensor, we have

$$
\gamma_{i-1}\left[\frac{t^{x}}{\Delta}+\tilde{\kappa}\left(s^{x}-1\right)\right]_{i-1}+\left[\gamma \frac{t^{x}}{\Delta}\right]_{j-1 / 2}=0
$$

or, in the case of constant $\gamma$

$$
t_{i-1}^{x}+t_{j-1 / 2}^{x}+\tilde{\kappa}_{i-1}\left(s_{i-1}^{x}-1\right) \Delta=0
$$

This relation between tangents, intersection position and curvature is the discrete equivalent of the Frenet-Serret relation (for constant $\kappa$ )

$$
\begin{aligned}
\oint_{A}^{B} d \boldsymbol{t} & =\oint_{A}^{B} \kappa \boldsymbol{n} d s \\
\left(\boldsymbol{t}_{B}-\boldsymbol{t}_{A}\right) \cdot \boldsymbol{x} & =\kappa\left(\oint_{A}^{B} \boldsymbol{n} d s\right) \cdot \boldsymbol{x} \\
t_{B}^{x}-t_{A}^{x} & =\kappa|B C|
\end{aligned}
$$

Note that it is not trivial that this relation is verified discretely. 


\section{Bibliography}

[1] T. Abadie, J. Aubin and D. Legendre. On the combined effects of surface tension force calculation and interface advection on spurious currents within volume of fluid and level set frameworks. Journal of Computational Physics, 297:611-636, 2015.

[2] M.O. Abu-Al-Saud, A. Riaz and H.A. Tchelepi. Multiscale level-set method for accurate modeling of immiscible two-phase flow with deposited thin films on solid surfaces. Journal of Computational Physics, :0, 2016.

[3] J.U. Brackbill, D.B. Kothe and C. Zemach. A continuum method for modeling surface tension. Journal of Computational Physics, 100(2):335-354, 1992.

[4] P.T. Brady, M. Herrmann and J.M. Lopez. Confined thermocapillary motion of a three-dimensional deformable drop. Physics of Fluids, 23(2):22101, 2011.

[5] A.J. Chorin. A numerical method for solving incompressible viscous flow problems. Journal of Computational Physics, 2(1):12-26, 1967.

[6] M. Detrixhe and T.D. Aslam. From level set to volume of fluid and back again at second-order accuracy. International Journal for Numerical Methods in Fluids, 80(4):231-255, 2016.

[7] A. du Chéné, C. Min and F. Gibou. Second-order accurate computation of curvatures in a level set framework using novel high-order reinitialization schemes. Journal of Scientific Computing, 35(23):114-131, 2008.

[8] M. Gorokhovski and M. Herrmann. Modeling primary atomization. Annu. Rev. Fluid Mech., 40:343366, 2008.

[9] D. Gueyffier, J. Li, A. Nadim, R. Scardovelli and S. Zaleski. Volume-of-fluid interface tracking with smoothed surface stress methods for three-dimensional flows. Journal of Computational physics, 152(2):423-456, 1999.

[10] M. Herrmann, J.M. Lopez, P. Brady and M. Raessi. Thermocapillary motion of deformable drops and bubbles. In Proceedings of the Summer Program, page 155. 2008.

[11] G.-S. Jiang and C.-W. Shu. Efficient implementation of weighted ENO schemes. Journal of Computational Physics, 126(1):202-228, 1996.

[12] A.R. Kovscek and C.J. Radke. Fundamentals of foam transport in porous media. ACS Publications, 1994.

[13] R. J. Leveque and Z. L. Li. The immersed interface method for elliptic-equations with discontinuous coefficients and singular sources. SIAM J. Numer. Anal., 31:1019, 1994.

[14] F. Luddens, M. Bergmann and L. Weynans. Enablers for high-order level set methods in fluid mechanics. International Journal for Numerical Methods in Fluids, 79(12):654-675, 2015. Fld.4070.

[15] L.W. Lake. Enhanced oil recovery. Prentice Hall, New Jersey, 1st edition, 1989.

[16] H. Lamb. Hydrodynamics. University Press, 1916.

[17] C. Ma and D. Bothe. Direct numerical simulation of thermocapillary flow based on the volume of fluid method. International Journal of Multiphase Flow, 37(9):1045-1058, 2011.

[18] C. Min. On reinitializing level set functions. Journal of Computational Physics, 229(8):2764-2772, 2010 .

[19] V. Miralles, A. Huerre, H. Williams, B. Fournié and M.-C. Jullien. A versatile technology for dropletbased microfluidics: thermomechanical actuation. Lab on a Chip, 15(9):2133-2139, 2015.

[20] M. Muradoglu and G. Tryggvason. A front-tracking method for computation of interfacial flows with soluble surfactants. Journal of computational physics, 227(4):2238-2262, 2008.

[21] C.S. Peskin. Flow patterns around heart valves: a numerical method. Journal of computational physics, 10(2):252-271, 1972.

[22] S. Popinet. An accurate adaptive solver for surface-tension-driven interfacial flows. Journal of Computational Physics, 228(16):5838-5866, 2009.

[23] S. Popinet. Numerical models of surface tension. Annual Review of Fluid Mechanics, 50(1):49-75, 2018.

[24] S. Popinet and S. Zaleski. A front-tracking algorithm for accurate representation of surface tension. International Journal for Numerical Methods in Fluids, 30(6):775-793, 1999.

[25] Y. Renardy and M. Renardy. Prost: a parabolic reconstruction of surface tension for the volume-offluid method. Journal of Computational Physics, 183(2):400-421, 2002.

[26] G. Russo and P. Smereka. A remark on computing distance functions. Journal of Computational Physics, 163(1):51-67, 2000.

[27] I. Seric, S. Afkhami and L. Kondic. Direct numerical simulation of variable surface tension flows 
using a volume-of-fluid method. Journal of Computational Physics, 352:615-636, 2018.

[28] N.O. Young, J.S. Goldstein and M.J. Block. The motion of bubbles in a vertical temperature gradient. Journal of Fluid Mechanics, 6(3):350-356, 1959. 